\title{
Illuminating the Opponent Process: Cocaine Effects on Habenulomesencephalic Circuitry
}

\author{
Patrick E. Rothwell and Stephan Lammel \\ Nancy Pritzker Laboratory, Department of Psychiatry and Behavioral Sciences, Stanford University, Stanford, California 94305 \\ Review of Jhou et al.
}

Drugs of abuse have profound effects on neural circuits that control emotional and motivational states. Most abused drugs promote dopaminergic signaling in pathways that originate in the ventral tegmental area (VTA), and these effects contribute to drug reward and reinforcement. As the initial positive effects of abused drugs dissipate, they are often followed by delayed negative effects, including states of anxiety and aversion associated with drug withdrawal. Classic "opponent process" theory suggests these delayed negative effects are a homeostatic response aimed at returning emotional state to equilibrium (Solomon and Corbit, 1974). The desire to alleviate these negative effects may motivate further drug use, leading to compulsive patterns of drug abuse associated with addiction (Koob and Le Moal, 2008).

Opponent process theory has had a major influence on the way we think about drug addiction, but underlying neurobiological mechanisms have proven surprisingly difficult to identify. Activation of VTA dopamine projections is sufficient to cause some emotional consequences of withdrawal (Radke et al., 2011), but the neural circuits engaged by dopamine that medi-

Received June 23, 2013; revised July 23, 2013; accepted July 25, 2013.

This work was supported by a fellowship from the National Institutes of Health (MH096491 to P.E.R.)

Correspondence should be addressed to either Patrick E. Rothwell or Stephan Lammel, Nancy Pritzker Laboratory, Department of Psychiatry and Behavioral Sciences, Stanford University School of Medicine, 265 Campus Drive, Stanford, CA 94305. E-mail: prothwel@stanford.edu or lammel@stanford.edu.

DOI:10.1523/JNEUROSCI.2680-13.2013

Copyright $\odot 2013$ the authors $\quad 0270-6474 / 13 / 3313935-03 \$ 15.00 / 0$ ate the opponent process remain poorly characterized. One potential candidate is the lateral habenula ( $\mathrm{LHb})$, which exerts indirect inhibitory control over VTA dopamine neurons through a GABAergic relay in the rostromedial tegmental nucleus (RMTg) (Barrot et al., 2012). This habenulomesencephalic circuit contributes to the generation of aversive behavioral states (Lammel et al., 2012), and a recent report in The Journal of Neuroscience (Jhou et al., 2013) shows cocaine affects this circuit in a manner consistent with opponent process theory.

Jhou and colleagues (2013) used an impressive array of electrophysiological, anatomical, behavioral, and optogenetic methods to study how cocaine influences the activity of neurons in LHb and RMTg. They first determined how $\mathrm{LHb}$ neurons respond to cocaine and dopamine, using both in vivo single-unit recordings in awake behaving rats and ex vivo whole-cell recordings in acute brain slices. Approximately half of $\mathrm{LHb}$ neurons were inhibited in the presence of cocaine or dopamine (Fig. 1A), showing reduced spiking and inhibitory outward currents, but a subset of these neurons subsequently exhibited "rebound" activation many minutes later (Fig. 1B). This biphasic temporal pattern of $\mathrm{LHb}$ neural activity mirrors the time course of cocaine's behavioral effects in a place-conditioning assay (i.e., initial reward followed by delayed aversion), which have been previously reported (Ettenberg, 2004) and were replicated by Jhou et al. (2013).
To further examine the role of habenulomesencephalic circuits in the opponent process actions of cocaine, Jhou et al. (2013) used a second behavioral assay in which rats receive an intravenous infusion of cocaine after running down a long alley. In this runway self-administration task, rats take longer and longer to reach the end of the alley over repeated daily trials, and become more likely to reverse direction and run away before receiving cocaine. This pattern of behavior is thought to emerge because the aversive effects of cocaine encourage avoidance responses (Ettenberg, 2004). Jhou et al. (2013) suggest that these avoidance responses depend on habenulomesencephalic circuitry, because rats run the alley more quickly and directly to receive cocaine following lesions of either the RMTg itself or the axonal projections from LHb to RMTg. Jhou et al. (2013) also shut down RMTg activity at different time points after cocaine infusion by injecting viruses expressing archaerhodopsin, a light-activated proton pump that hyperpolarizes neurons. Optogenetic inhibition of the RMTg effectively prevented avoidance responses in the runway self-administration task, but only when applied 15-25 min after cocaine infusion, the time at which some LHb neurons exhibit rebound excitation and cocaine becomes aversive (Fig. 1B). In contrast, optogenetic inhibition of the RMTg had no effect on avoidance responses in the runway self-administration task when applied for 10 min immediately after cocaine infusion.

A notable aspect of the data reported by Jhou et al. (2013) is the heterogeneity 
of responses recorded in $\mathrm{LHb}$ neurons. Of the subpopulation $(\sim 50 \%)$ that was initially inhibited by cocaine or dopamine application, only a subset $(\sim 30-40 \%)$ of LHb neurons exhibited a biphasic response with subsequent rebound activation. One plausible explanation for this heterogeneity is that $\mathrm{LHb}$ neurons projecting to different downstream targets are regulated by cocaine in a pathway-specific fashion (Maroteaux and Mameli, 2012). Jhou et al. (2013) investigated this possibility using retrograde tracers (cholera-toxin B and Neuro-DiI) to identify and differentiate LHb neurons that project either to the RMTg or directly to the VTA. Using c-Fos immunohistochemistry as an index of neuronal activation, they found that cocaine only activated $\mathrm{LHb}$ neurons that projected to the RMTg. To determine whether this population of LHb neurons exhibits a biphasic pattern of activity after cocaine exposure, Jhou et al. (2013) injected a retrograde tracer into the RMTg, thus labeling $\mathrm{LHb}$ neurons that project to this site and permitting targeted recordings in brain slices from this specific population of cells. Surprisingly, of eight neurons labeled and recorded in this fashion, only two (25\%) exhibited rebound activation following dopamine application. Thus, projection to the RMTg does not appear to be the primary factor dictating whether $\mathrm{LHb}$ neurons exhibit a biphasic response to cocaine, as heterogeneous responses are observed even within this anatomically defined population of neurons.

Though only a subset of LHb neurons projecting to RMTg exhibit biphasic responses to cocaine, this subpopulation may be particularly important for mediating the delayed aversive effects of cocaine, as indicated by the behavioral and immunohistochemical data presented by Jhou et al. (2013). However, these data encourage further consideration of the potential function of $\mathrm{LHb}$ neurons sending axons to areas other than RMTg, which may also exhibit biphasic responses to cocaine. Although the direct projection from $\mathrm{LHb}$ to VTA is not affected by cocaine (Maroteaux and Mameli, 2012; Jhou et al., 2013), LHb neurons also project to a variety of other targets (Araki et al., 1988), including the dorsal and medial raphe nuclei and the laterodorsal tegmentum (LDT). The LDT is a particularly interesting target given its projection to the VTA, which mediates rewarding behavioral states (Lammel et al., 2012) as well as behavioral responses to psychostimulant drugs (Nelson et al., 2007). The effects of cocaine on these other LHb output pathways will be an interesting topic for future research (Fig. 1), and
A

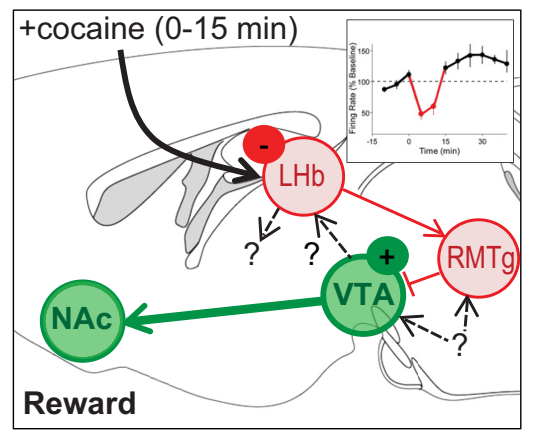

B

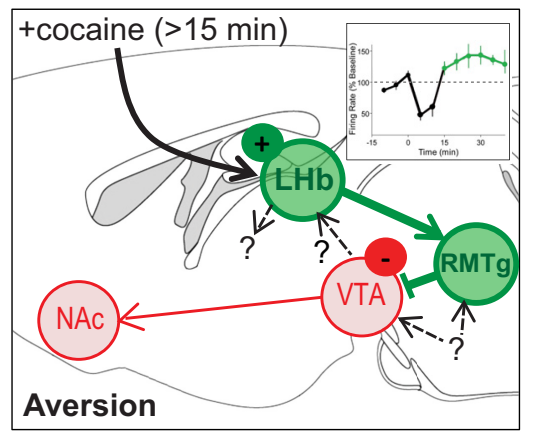

Figure 1. Proposed model for the acute rewarding and delayed aversive effects of cocaine through drug actions on habenulomesencephalic circuitry. Green circles indicate brain areas with increased neuronal activity; red circles correspond to decreased neuronal activity. $\boldsymbol{A}$, Immediately after cocaine infusion, inhibition of a subset of neurons in the LHb and RMTg results in disinhibition of VTA dopamine neurons projecting to nucleus accumbens (NAc), generating a rewarding behavioral state. $\boldsymbol{B}$, Fifteen minutes after cocaine infusion, rebound activation of a subpopulation of $L H b$ and RMTg neurons causes inhibition of dopamine neurons projecting to NAc, generating an aversive behavioral state. Insets in $\boldsymbol{A}$ and $\boldsymbol{B}$ show firing rate of $L H b$ neurons. Question marks indicate several avenues for future research: characterizing the neurons that project from VTA to $L H b$, examining $L H b$ projections to areas other than RMTg, and identifying nonhabenular inputs to VTA and RMTg (such as those from the extended amygdala).

may further explain the heterogeneity of $\mathrm{LHb}$ responses to cocaine reported by Jhou et al. (2013).

It will also be important to more clearly define the specific populations of dopamine neurons that project to $\mathrm{LHb}$ (Fig. 1). The midbrain dopamine system contains distinct subpopulations of dopamine neurons with different anatomical and physiological properties, defined by their synaptic inputs and axonal projection targets (Lammel et al., 2012). Dopamine neurons that project to $\mathrm{LHb}$ are located in the anterior VTA (Gruber et al., 2007), but their electrophysiological properties have not been characterized, and the behavioral consequence of manipulating these mesohabenular dopamine neurons remains to be determined. It is also worth noting that $\sim 50 \%$ of the VTA neurons that project to $\mathrm{LHb}$ do not express tyrosine hydroxylase, a canonical marker for dopaminergic neurons (Gruber et al., 2007). The potential function of nondopaminergic mesohabenular projections should be addressed in future research.

Another direction for future research involves characterization of nonhabenular inputs to the RMTg and VTA that may also contribute to the opponent process action of cocaine and other drugs (Fig. 1). For example, the RMTg and VTA receive projections from portions of the extended amygdala, including the amygdala itself, the nucleus accumbens shell, and the bed nucleus of the stria terminalis (Jhou et al., 2009; Barrot et al., 2012). Extended amygdala circuits contribute to a variety of aversive behavioral states and play a central role in the negative emotional con- sequences of drug withdrawal (Koob and Le Moal, 2008). Importantly, dopamine is released in the extended amygdala after exposure to a variety of abused drugs (Carboni et al., 2000), providing a mechanism for engaging these structures in the opponent process. Thus, extended amygdala projections to the midbrain may function in a parallel and complementary fashion to the habenulomesencephalic circuit described by Jhou et al. (2013).

A particularly intriguing implication of this research is that habenulomesencephalic circuitry may contribute to opponent process actions of multiple classes of abused drugs. The activity of neurons in the RMTg and their inhibitory synapses on dopamine neurons are negatively regulated by opioids and cannabinoids (Barrot et al., 2012). While rebound activation of RMTg neurons has not been reported to occur following acute exposure to these drugs, repeated drug exposure may cause functional changes in habenulomesencephalic circuitry (Maroteaux and Mameli, 2012), perhaps contributing to the increasing strength of the opponent process that develops over the course of chronic drug use (Solomon and Corbit, 1974; Koob and Le Moal, 2008). Because opponent process mechanisms are thought to play a general role in addiction to many different kinds of drugs, it is tantalizing to speculate the habenulomesencephalic circuit may represent a central conduit for triggering compulsive patterns of drug abuse.

\section{References}

Araki M, McGeer PL, Kimura H (1988) The efferent projections of the rat lateral habenular 
nucleus revealed by the PHA-L anterograde tracing method. Brain Res 441:319-330. CrossRef Medline

Barrot M, Sesack SR, Georges F, Pistis M, Hong S, Jhou TC (2012) Braking dopamine systems: a new GABA master structure for mesolimbic and nigrostriatal functions. J Neurosci 32:14094-14101. CrossRef Medline

Carboni E, Silvagni A, Rolando MT, Di Chiara G (2000) Stimulation of in vivo dopamine transmission in the bed nucleus of stria terminalis by reinforcing drugs. J Neurosci 20: RC102. Medline

Ettenberg A (2004) Opponent process properties of self-administered cocaine. Neurosci Biobehav Rev 27:721-728. CrossRef Medline

Gruber C, Kahl A, Lebenheim L, Kowski A, Dittgen A, Veh RW (2007) Dopaminergic projections from the VTA substantially contribute to the mesohabenular pathway in the rat. Neurosci Lett 427:165-170. CrossRef Medline

Jhou TC, Geisler S, Marinelli M, Degarmo BA, Zahm DS (2009) The mesopontine rostromedial tegmental nucleus: a structure targeted by the lateral habenula that projects to the ventral tegmental area of Tsai and substantia nigra compacta. J Comp Neurol 513:566-596. CrossRef Medline

Jhou TC, Good CH, Rowley CS, Xu SP, Wang H, Burnham NW, Hoffman AF, Lupica CR, Ikemoto S (2013) Cocaine drives aversive conditioning via delayed activation of dopamineresponsive habenular and midbrain pathways. J Neurosci 33:7501-7512. CrossRef Medline

Koob GF, Le Moal M (2008) Addiction and the brain antireward system. Annu Rev Psychol 59:29-53. CrossRef Medline

Lammel S, Lim BK, Ran C, Huang KW, Betley MJ, Tye KM, Deisseroth K, Malenka RC
(2012) Input-specific control of reward and aversion in the ventral tegmental area. Nature 491:212-217. CrossRef Medline

Maroteaux M, Mameli M (2012) Cocaine evokes projection-specific synaptic plasticity of lateral habenula neurons. J Neurosci 32:12641-12646. CrossRef Medline

Nelson CL, Wetter JB, Milovanovic M, Wolf ME (2007) The laterodorsal tegmentum contributes to behavioral sensitization to amphetamine. Neuroscience 146:41-49. CrossRef Medline

Radke AK, Rothwell PE, Gewirtz JC (2011) An anatomical basis for opponent process mechanisms of opiate withdrawal. J Neurosci 31: 7533-7539. CrossRef Medline

Solomon RL, Corbit JD (1974) An opponentprocess theory of motivation. I. Temporal dynamics of affect. Psychol Rev 81:119-145. CrossRef Medline 